\title{
Revisión del estado del arte de sistemas DX-SAHP para la obtención de agua caliente sanitaria
}

\section{(Review of the state of art of DX-SAHP systems to obtain domestic hot water)}

\author{
William Quitiaquez, ${ }^{1}$ Isaac Simbaña, ${ }^{1}$ C. A. Isaza-Roldán, ${ }^{2}$ Patricio Quitiaquez, ${ }^{1}$ César Nieto-Londoño, ${ }^{2}$ \\ Fernando Toapanta-Ramos ${ }^{1}$
}

\begin{abstract}
Resumen
El objetivo del presente artículo es revisar de forma detallada las distintas investigaciones realizadas con sistemas de bombas de calor de expansión directa asistidas por energía solar (DX-SAHP) utilizadas para el calentamiento de agua en los últimos años. El creciente consumo energético, el uso de refrigerantes que debilitan la capa de ozono, la emisión de gases de efecto invernadero a la atmósfera y los efectos en el calentamiento global, son los principales problemas que presentan los sistemas convencionales de calentamiento de agua. El uso de hidrocarburos como refrigerantes representa una reducción en la contaminación, además de ser una de las mejores opciones para reemplazar a los clorofluorocarbonos e hidroclorofluorocarbonos por sus valores de potencial de calentamiento global y potencial de destrucción de la capa de ozono cercanos a 0. Un sistema DX-SAHP aprovecha la energía solar térmica directamente, empleando un colector solar de placa plana sin cubierta. Esos sistemas proporcionan agua caliente sanitaria a más de $50{ }^{\circ} \mathrm{C}$, calentando volúmenes de agua de hasta 200 litros, alcanzando valores de COP superiores a 4. La energía solar y el uso de refrigerantes alternativos de bajo impacto ambiental son propuestos para alcanzar este propósito.
\end{abstract}

\section{Palabras clave}

Bombas de calor; refrigerantes alternativos; energía solar; DX-SAHP; colector solar.

\begin{abstract}
The investigation purpose is to elaborate a detailed review about previous published investigations with direct-expansion solar-assisted heat pumps (DX-SAHP) used for water heating, in the last years. The growing energy consumption, the using of refrigerants that weaken the ozone layer, the greenhouse gases emission to the atmosphere and the global warming effect are the main problems that the conventional water heating systems present. Using hydrocarbons as refrigerants represents a pollution reduction and it is also the best option to replace hydrochlorofluorocarbons and chlorofluorocarbons, due to their low values of global warming potential and ozone depletion potential, close to 0. A DX-SAHP system takes advantage of the thermal solar energy directly by using a bare flat-plate solar collector. These systems provide domestic hot water over $50^{\circ} \mathrm{C}$ and heating water volumes until 200 liters reaching COP values higher than 4 . Solar energy and the using of alternative refrigerants with a low environmental impact are proposed to reach this purpose.
\end{abstract}

\section{Keywords}

Heat pumps; alternative refrigerants; solar energy; DX-SAHP; solar collector.

\section{Introducción}

Kannan y Vakeesan (2016) afirman que, en los últimos años, el consumo de energía primaria en el mundo ha aumentado debido a la explosión demográfica y a los avances tecnológicos. El acceso de la sociedad a la energía en el período comprendido entre los años 2005 y 2014 ha crecido a una tasa aproximada del $24 \%, 19 \%$ y $7 \%$ en los sectores industrial, transporte y residencial, respectivamente. El consumo de electricidad también se ha incrementado a una tasa 
aproximada del $35 \%$, $21 \%$ y $27 \%$ en los sectores antes mencionados (International Energy Agency, 2019; Zhou y Feng, 2016).

Para el Ministerio del Ambiente de Ecuador (2019), el Protocolo de Kioto promovió la reducción de las emisiones de gases de efecto invernadero (GEI) en 37 países industrializados y la Unión Europea. Gallo, Faccilongo y La Sala (2017) mencionan que el objetivo es reducir las emisiones de dióxido de carbono (CO2), metano (CH4) y óxido nitroso (N2O), así como tres gases industriales fluorados, hidrofluorocarbonos (HFC), perfluorocarbonos (PFC) y hexafluoruro de azufre (SF6). La reducción está estimada en un 5 \% anual, a partir de su declaración en 1990 (Miyamoto y Takeuchi, 2019). Esta información se la obtuvo de los 185 países participantes, así como de la Unión Europea en el período de 1990 hasta 2012 (Maamoun, 2019). Según la Secretaría Nacional de Planificación y Desarrollo de Ecuador (2013), el Protocolo de Montreal fue aprobado en septiembre de 1987, con el fin de controlar, limitar y regular, tanto el consumo como la producción de clorofluorocarbonos (CFCs), hidroclorofluorocarbonos (HCFCs) y halones, sustancias que agotan la capa de ozono, y así tratar de evitar daños a la salud y al medio ambiente. En estudios presentados por investigadores como Gorozabel, Chaturvedi y Almogbel (2005); Cerit y Erbay (2013); Chow et al. (2010), se utilizan refrigerantes HFC, el R134a, R410A y R407C, puesto que no destruyen la capa de ozono. En la actualidad, se presentan nuevas alternativas en refrigerantes, los hidrocarburos $(\mathrm{HC}$ ) junto con las hidrofluoroolefinas (HFO) que son refrigerantes de cuarta generación y que, en ciertos casos, presentan cero agotamiento de la capa de ozono. El Acuerdo de París, instaurado en 2015, también pretende mantener el aumento de la temperatura media mundial por debajo de $2^{\circ} \mathrm{C}$ (Bauer y Menrad, 2019). El grupo objetivo es el sector industrial, donde inicialmente se planteó que la emisión de gases de efecto invernadero no produzca un incremento mayor a $1.5^{\circ} \mathrm{C}$. Según Vargas et al. (2019) , la implementación de sistemas asistidos por energía solar minimizan las emisiones de C02 a la atmósfera ya que utilizan una fuente de energía amigable con el medio ambiente.

Las bombas de calor asistidas por energía solar (SAHP, por sus siglas en inglés) son sistemas que trabajan utilizando el ciclo termodinámico de refrigeración por compresión de vapor. Autores, como Buker y Riffat (2016), las definen como un tipo de bombas de calor en las que se integra un colector solar térmico que capta la radiación solar y que utiliza un refrigerante como fluido de trabajo. Los estudios están direccionados hacia la mejora de la eficiencia energética y como una opción para brindar un desarrollo sustentable, utilizando fuentes renovables de energía en lugar de combustibles fósiles (Shi, Aye, Li y Du, 2019). La primera propuesta para implementar un colector solar a estos sistemas data de 1976 por parte de Charters y Taylor (1976). Como datos preliminares, un calentador de agua eléctrico puede incurrir en un consumo diario de $5.5 \mathrm{~kW}$ (Homecenter, 2019), un calentador de agua a gas puede presentar un consumo de $6.27 \mathrm{~kW} \cdot \mathrm{h} /$ día (Haceb, 2019), mientras que un calentador de agua con bomba de calor tiene un consumo apenas de $1.3 \mathrm{~kW} \cdot \mathrm{h} /$ día (Junkers, 2019).

El objetivo de la investigación es realizar una revisión actual de las investigaciones sobre los sistemas de bomba de calor asistidos por energía solar (SAHP). Además, se presenta la información de la literatura relacionada a los recientes avances de rendimiento en sistemas de bombas de calor de expansión directa asistidas por energía solar (DX-SAHP, por sus siglas en inglés). Se ha considerado parámetros como el coeficiente de operación del sistema (COP), impactos ambientales producidos por el uso de refrigerantes dañinos con el ambiente, geometría y área de los colectores solares, materiales utilizados, temperatura del agua y eficiencia del colector. Este documento se encuentra distribuido de la siguiente manera. La Introducción presenta la justifi- 
cación e importancia de la realización de esta investigación, revisando investigaciones relacionadas al calentamiento de agua en el sector residencial utilizando sistemas SAHP. Luego se detalla la descripción de los sistemas SAHP, su clasificación particularizando en la configuración de DXSAHP, el colector/evaporador que es el elemento principal en estos sistemas, los refrigerantes utilizados y el coeficiente de rendimiento. Finalmente, las conclusiones discuten los puntos más relevantes del documento para presentar información a considerar en futuras investigaciones.

\section{Descripción de los sistemas SAHP}

Una bomba de calor asistida por energía solar se caracteriza por utilizar la radiación emitida por el Sol para su funcionamiento, esto provoca una reducción en el uso de combustibles fósiles y en las emisiones de $\mathrm{CO}_{2}$ a la atmósfera. Un sistema SAHP utiliza un fluido de trabajo que puede ser agua o un refrigerante. Cuando la radiación solar incide sobre la superficie del colector/evaporador se presenta una transferencia de calor desde el exterior del tubo o placa hacia el fluido. Esto provoca una elevación de temperatura y en caso de utilizar un refrigerante, este cambia de fase líquida a vapor (Buker y Riffat, 2016). Un sistema de tipo bomba de calor presenta varias aplicaciones, una de ellas es la generación de agua caliente, disminuyendo la demanda energética. Según Duarte, Paulino, Pabón, Sawalha y Machado (2019), al utilizar una fuente renovable, como es la energía solar, se aprovecha la energía térmica y, mediante la aplicación de los mecanismos de transferencia de calor, la transmite al fluido.

Las bombas de calor pueden extraer el calor de diferentes fuentes, del suelo, aire, fuentes de agua y son utilizadas para el calentamiento de espacios o de agua (Deng y Yu, 2016). En función del modo de conexión del colector /evaporador, para Cai, Ji, Wang y Huang (2016), los sistemas SAHP se presentan en dos modelos, bombas de calor de expansión directa asistidas por energía solar y en bombas de calor de expansión indirecta asistidas por energía solar.

\section{Bombas de calor de expansión directa asistidas por energía solar (DX-SAHP)}

En un sistema DX-SAHP, el colector solar y el evaporador de la bomba de calor se integran en una sola unidad para transferir la energía solar al refrigerante. Cai, Li, Ji y Zhou (2019) indican que la implementación de estos sistemas se centra en el sector residencial, tanto para aire acondicionado, como para calentamiento de espacios y agua, debido a su bajo consumo energético. Los primeros investigadores que reportan estudios con bombas de calor asistidas por energía solar fueron Sporn y Ambrose (1955). Presentaron resultados relevantes, pero no logran demostrar el potencial de un sistema DX-SAHP debido a un aparente desajuste entre la capacidad de bombeo de flujo másico del compresor y la capacidad de evaporación del colector (Gorozabel y Carbonell, 2016). Los sistemas SAHP trabajan en un ciclo de refrigeración por compresión de vapor y constan de cuatro componentes principales, el evaporador, compresor, condensador y un dispositivo de expansión, como se esquematiza en la figura 1 (Wang y Ge, 2016).

El funcionamiento de los sistemas DX-SAHP inicia cuando el refrigerante absorbe el calor producto de la energía solar incidente y del ambiente. Kalogirou (2014) explica que esta energía térmica se transforma en calor y puede almacenarse o utilizarse directamente. Si se utiliza directamente, el fluido de trabajo se evapora en el colector/evaporador, luego se comprime en un compresor hermético recíproco para convertirse en vapor sobrecalentado de alta temperatura y alta presión. El vapor ingresa en el condensador que actúa como intercambiador de calor entre el refrigerante y el medio en donde se condensa. 
Figura 1. Diagrama esquemático de un sistema DX-SAHP (Wang y Ge, 2016)

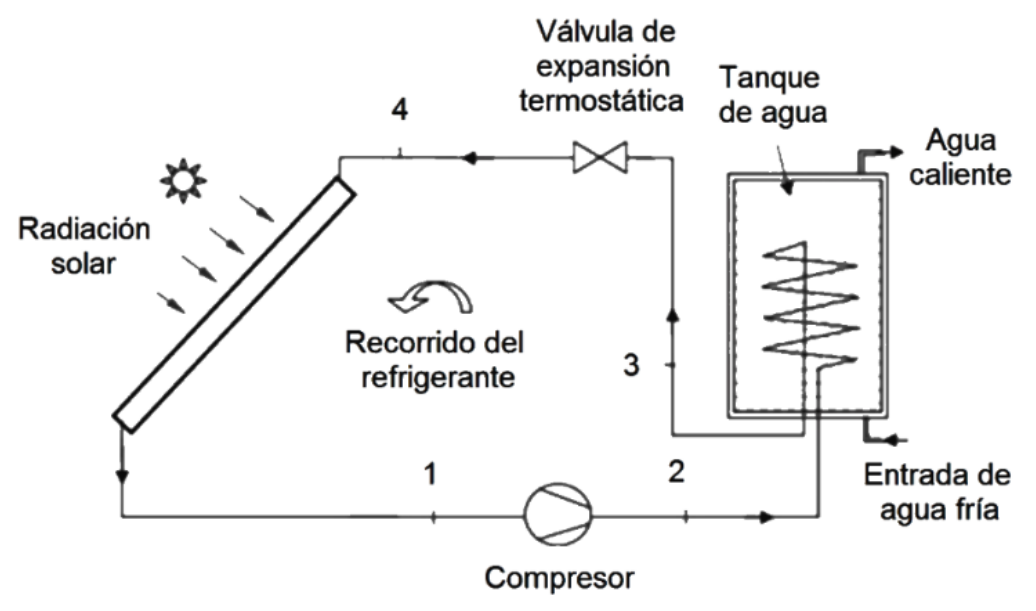

El calor rechazado en este procedimiento es absorbido por el medio, generalmente agua contenida en un tanque de almacenamiento. Posteriormente, el refrigerante pasa por una válvula de expansión donde se expande irreversible y adiabáticamente. Para finalizar, el refrigerante fluye hacia el colector/evaporador y se evapora por la absorción de energía térmica (Huang, Ji, Xu y Li, 2016). El diagrama temperatura vs. entropía (T-s) de un ciclo ideal de refrigeración por compresión de vapor se muestra en la figura 2.

Figura 2. Diagrama T-s para un sistema SAHP (Malali, Chaturvedi y Abdel-Salam, 2016)

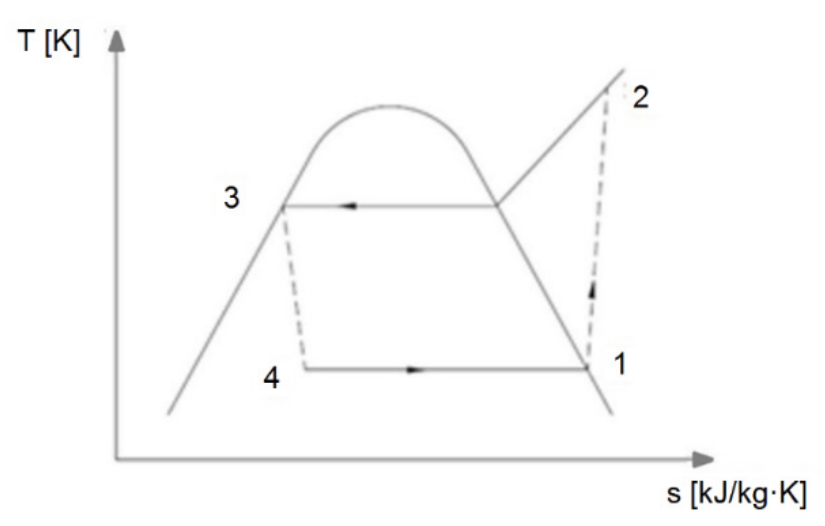

El uso de los sistemas DX-SAHP conlleva varias ventajas, principalmente el no utilizar combustibles provenientes de fuentes fósiles. El tamaño es menor en comparación con un sistema de calentamiento de agua a gas y no necesita ser colocado en un lugar abierto para evitar fugas de gas o posibles incidentes que afecten a la integridad de las personas. Al ser equipos que utilizan una fuente renovable de energía, como es la energía solar, para Gadea y Gonzalo (2019) la contaminación y las emisiones de gases de efecto invernadero a la atmósfera disminuyen. El colector/evaporador puede ser colocado en cualquier lugar para que pueda funcionar, de preferencia, debería ser colocado en un lugar libre de sombras para que la radiación solar incida directamente sobre él (Sheu y Mitsos, 2013). Krockenberger, DeGrove, Hutzel y Foreman (2014) 
afirman que la combinación de dos sistemas, una bomba de calor y la energía solar, reducen los costos de energía y aumentan la eficiencia. Mediante el uso de unidades motrices de velocidad variable se obtiene un mejor desempeño. Cengel y Boles (2015) explican que, al implementar un compresor de velocidad variable, se permite que la unidad opere a su máxima eficiencia para distintas necesidades de calentamiento y enfriamiento en determinadas condiciones atmosféricas. Shi, Aye, Li y Du (2019) han registrado investigaciones de sistemas DX-SAHP aproximadamente desde mediados del año 1970 y se localizan en las costas del Pacífico y zona sur europea. La Figura 3 indica los países con autores que han presentado estudios referentes al tema.

Figura 3. Países con investigaciones sobre sistemas DX-SAHP (Shi, Aye, Li y Du, 2019)

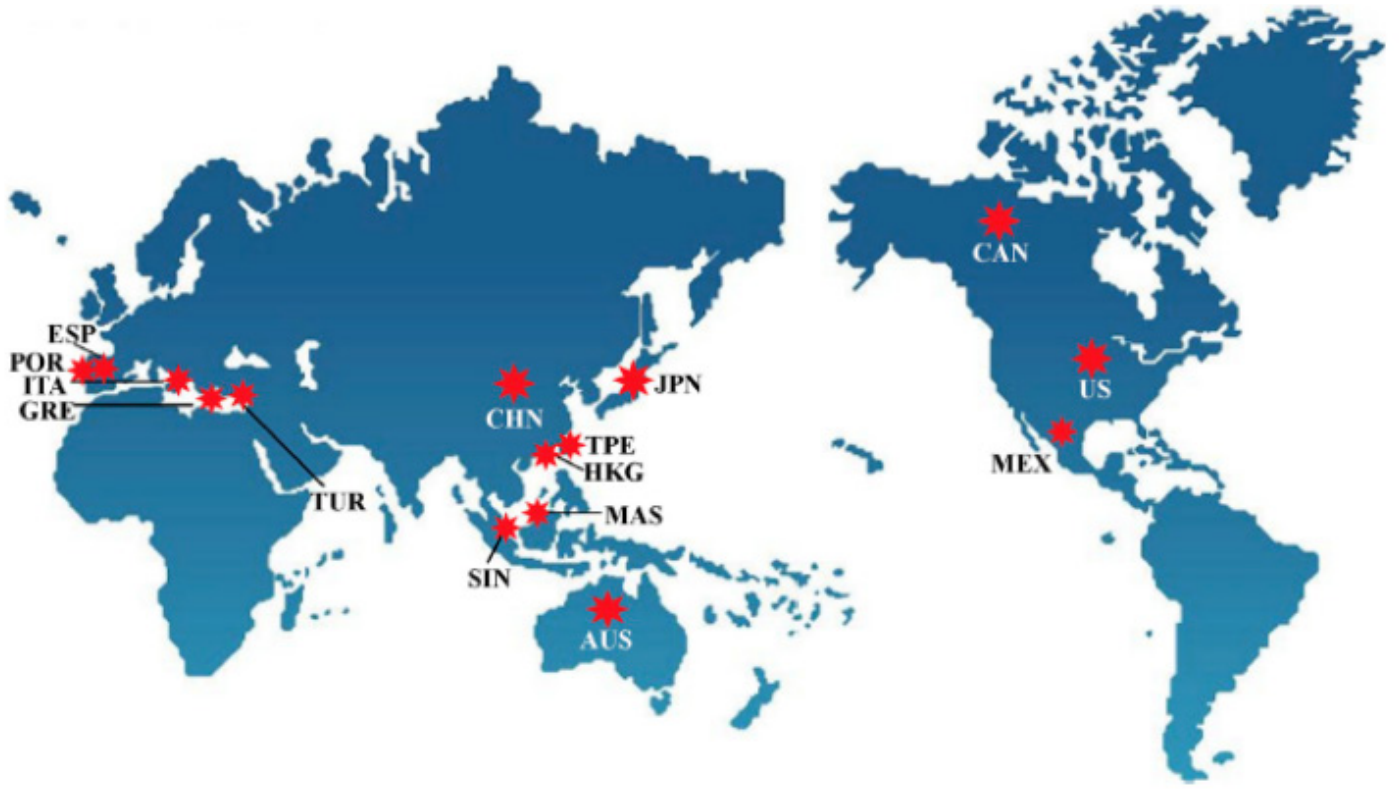

Investigadores a nivel mundial, como Buker y Riffat (2016), Tagliafico, Scarpa y Valsuani (2014), Hossain et al. (2011), han realizado diferentes investigaciones relacionadas a sistemas DX-SAHP. A continuación, se presenta una revisión de la literatura relacionada a los mencionados sistemas, un listado comprendido con investigaciones desde 1955, año en el cual Sporn y Ambrose (1955) muestran una investigación sobre la bomba de calor y la energía solar.

\section{Colector/evaporador}

Buker y Riffat (2016) define un colector solar como un elemento que transforma la energía solar incidente en forma de calor y la transmite al fluido que circula en su interior. Los colectores solares de placa plana son dispositivos que captan la energía solar térmica la cual puede ser utilizada en aplicaciones como calefacción, calentamiento de agua, calefacción de habitaciones e incluso aplicaciones industriales (Pandey y Chaurasiya, 2017). Se pueden generar varios diseños, uno de ellos es en forma de serpentín, modelo que no presenta el problema de distribución desigual del flujo en los diversos tubos (Kalogirou, 2014). El diseño de la placa contempla dos opciones, cuando se presenta una sola lámina en la que están colocados todos los canales, como se muestra en la Figura 4a. La segunda opción se presenta en la Figura 4b, cuando cada 
uno de los tubos son montados en cuerpos separados. En función de su estructura, Zhu, Xie, Zhang y Guan (2013) clasifican a los colectores/evaporadores en colectores de placa desnuda, sin cubierta o de tipo roll bond, colectores de placa de vidrio con cubierta y colectores/evaporadores dobles (un colector de placa desnuda y un colector de placa con cubierta).

Figura 4. Colector solar de placa plana a. sin cubierta b. cubierto (Hossain et al., 2011; Kalogirou, 2014).

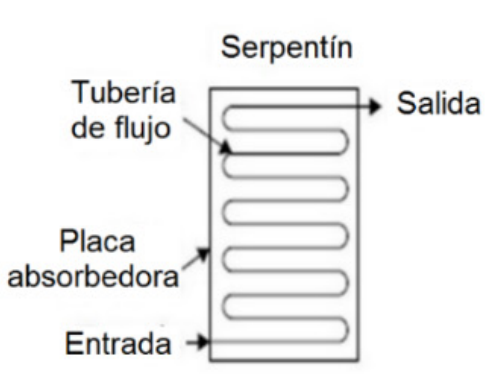

a.

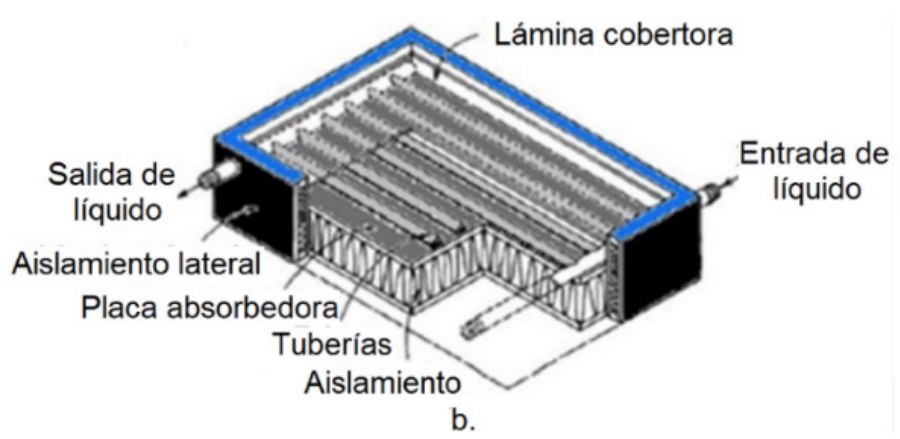

Según Hossain et al. (2011), los colectores solares se pueden clasificar en colectores de concentración y de placa plana los cuales se pueden utilizar en los sistemas DX-SAHP. Estos colectores son los más utilizados para el calentamiento de agua en el sector residencial. Para la eficiencia de los colectores solares, se tiene aproximadamente un error de $4 \%$ entre los valores experimentales y los valores obtenidos mediante modelado y simulación (Benavides, Jurado y González, 2018). En la Figura 5 se presenta un diagrama esquemático del flujo de calor a través de un colector de placa plana con cubierta, el cual absorbe aproximadamente un $80 \%$ de la energía solar térmica.

Figura 5. Flujo de calor en un colector solar de placa plana (Hossain et al., 2011).

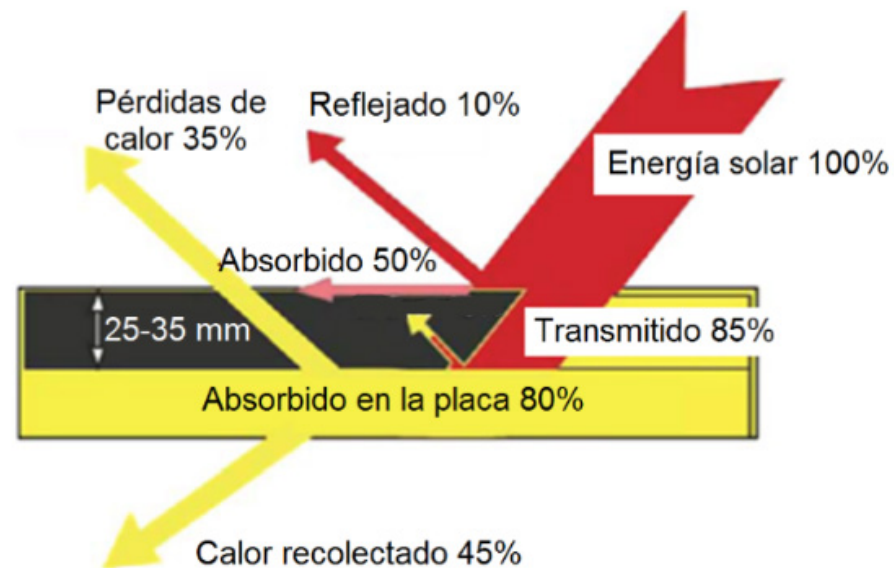

El diseño de este tipo de colectores está en un constante desarrollo tecnológico, ya que las condiciones climáticas influyen en la conductividad térmica hacia el refrigerante (Velasco, Goyos, Delgado y Freire, 2016). A continuación, se presenta una revisión de investigaciones que 
han considerado el uso de colectores/evaporadores de diferentes tipos. Huang, Ji, Xu y Li (2016) utilizaron un sistema DX-SAHP con colectores solares sin cubierta para la calefacción de un espacio. El equipo trabaja a una temperatura ambiente que oscila entre 3 y $7^{\circ} \mathrm{C}$, con humedades relativas de 50,70 y $90 \%$ y las condiciones de radiación solar que se presentaron en las pruebas realizadas son $0,100,200,300 \mathrm{~W} / \mathrm{m}^{2}$, respectivamente. De los resultados obtenidos, los autores manifiestan que una radiación de $100 \mathrm{~W} / \mathrm{m}^{2}$ ayuda a prevenir la formación de hielo cuando la temperatura ambiente es superior a $3^{\circ} \mathrm{C}$ y la humedad relativa es del $70 \%$. En función de la radiación solar, el sistema puede mejorar o reducir el rendimiento. El coeficiente de operación del sistema en un tiempo de 90 minutos fue aproximadamente 2.4 para una temperatura ambiente de $5^{\circ} \mathrm{C}$ y una humedad relativa de $70{ }^{\circ} \mathrm{C}$, radiación solar de $300 \mathrm{~W} / \mathrm{m}^{2}$. Mientras que a una radiación solar a $100 \mathrm{~W} / \mathrm{m}^{2}$, el COP fue 1.8 manteniendo los mismos valores. Una suposición que utilizan los autores es que la temperatura de evaporación y de condensación son -5 y $55^{\circ} \mathrm{C}$, respectivamente. La eficiencia exergética alcanzada por el sistema es de $58.48,59.16$ y $23.81 \%$ para el compresor, condensador y colector solar, respectivamente.

Aguilar, Aledo y Quiles (2016) analizaron la opción de combinar paneles solares fotovoltaicos junto a bombas de calor para la producción de agua caliente sanitaria (ACS) en una residencia promedio de 4 personas. Los resultados presentados indican un consumo de 130 litros diarios a $55^{\circ} \mathrm{C}$, y una producción de $6.2 \mathrm{~kW} \cdot h$ en un día. Así, la contribución solar alcanzó un $60 \%$, con resultados desarrollados a lo largo de un año.

En la investigación realizada por Cerit y Erbay (2013), se utilizó aluminio como material para la fabricación de un colector/evaporador de tipo roll bond y el fluido de trabajo fue el refrigerante R134a. Se han investigado tres sistemas de bombas de calor asistidas por energía solar con un área de captación solar de $1 \mathrm{~m}^{2}$, cambiando el diseño del colector/evaporador, como se indica en la Figura 6. Los valores obtenidos del COP en los sistemas 1, 2 y 3 fueron 3.30, 3.14 y 2.42 , respectivamente, en las mismas condiciones de operación. La temperatura inicial del agua fue de $13^{\circ} \mathrm{C}$ a una temperatura ambiente máxima de $20^{\circ} \mathrm{C}$ y una intensidad de radiación solar de $536 \mathrm{~W} / \mathrm{m}^{2}$ al medio día.

Figura 6. Configuraciones realizadas en el evaporador sin cubierta a. Sistema 1 b. Sistema 2 c. Sistema 3 (Cerit y Erbay, 2013; Ravi, Krishnaiah, Akella y Azizuddin, 2015)

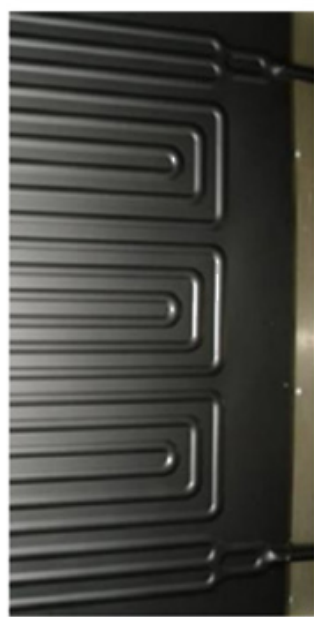

a.

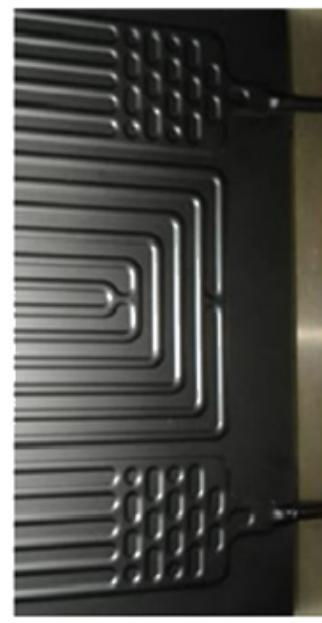

b.

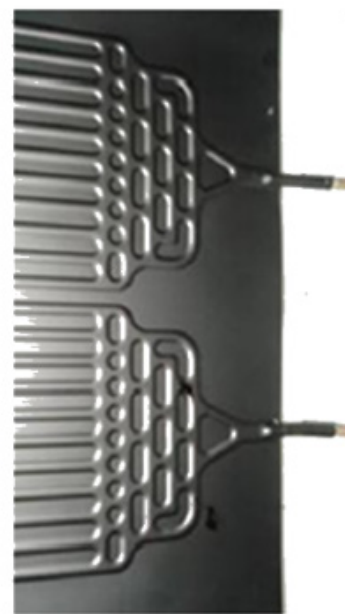

C. 
Según Calise, Dentice D`Accadia, Figaj y Vanoli (2016), los paneles solares fotovoltaicos pueden ser colocados en una bomba de calor de expansión directa asistida por energía solar y son denominados sistemas híbridos. Son colectores que producen energía eléctrica y térmica simultáneamente, aunque la energía es fluctuante debido a la radiación externa y a la temperatura. Colangelo, Favale, Miglietta y De Risi (2016) manifiestan que, cuando la radiación solar es alta, los sistemas fotovoltaicos funcionan en condiciones desfavorables, porque la eficiencia de conversión de energía de los módulos fotovoltaicos es inversamente proporcional a la temperatura de funcionamiento. Las altas temperaturas disminuyen el voltaje de los paneles a un valor reducido para cargar las baterías por completo.

Kong, Li, Lin y Yang (2017) realizaron una investigación sobre los sistemas solares del tipo bombas de calor con asistencia directa. Los componentes utilizados en la investigación fueron una válvula de expansión electrónica, un compresor hermético de tipo rotatorio que utiliza refrigerante R410A como fluido de trabajo. Un condensador tipo serpentín de diámetro $99 \times 0.75$ $\mathrm{mm}$ de longitud, que se encuentra inmerso dentro de un tanque de agua de 150 litros. El sistema consta de cuatro placas absorbentes de aluminio en paralelo con una superficie total de $4.2 \mathrm{~m}^{2}$. Los resultados obtenidos indican que el tiempo de calentamiento varía según la intensidad de la radiación solar. Además, el COP promedio a una velocidad de rotación del compresor de 1500 rpm fue $144.6 \%$ mayor que a 3300 rpm. Cuando la temperatura inicial del agua incrementa desde 5 hasta $30{ }^{\circ} \mathrm{C}$, el tiempo de calentamiento se reduce aproximadamente un 53.9\%. Los autores manifiestan que los refrigerantes $\mathrm{HC}$ poseen propiedades de transporte superiores al R22, además que el refrigerante R134a tiene la temperatura crítica más alta entre los refrigerantes que se consideran en el estudio.

Kuang y Wang (2006) realizaron una investigación sobre un sistema DX-SAHP que es utilizado para el calentamiento de espacios en invierno, aire acondicionado en verano y para el calentamiento de agua durante todo el año. Contiene un conjunto de 10 colectores de placa plana de tipo roll bond con un área superficial de $10.5 \mathrm{~m}^{2}$ orientados hacia el sur, considerando que la investigación fue realizada en China. Además, un tanque de almacenamiento de $1 \mathrm{~m}^{3}$, el fluido de trabajo fue el refrigerante R22 y un compresor de velocidad variable. Este sistema suministra 200 litros de agua caliente diarios, con una temperatura final del agua de $50^{\circ} \mathrm{C}$. El material de las placas colectoras es aluminio de $1 \mathrm{~mm}$ de espesor y fueron unidas de forma presurizada dejando un diámetro interior y un paso entre tubos de 8 y $40 \mathrm{~mm}$, respectivamente. El compresor hermético de tipo rotatorio tiene una potencia nominal de entrada de 3 hp y la variación de velocidad se logró a través de un variador de frecuencia (VFD) capaz de ajustarla de 30 a $90 \mathrm{~Hz}$. Así se puede evitar el desajuste entre la carga variable en el sistema y la capacidad constante del compresor.

Moreno-Rodríguez, García-Hernando, González-Gil e Izquierdo (2013) realizaron una investigación utilizando una DX-SAHP con un compresor de $1.1 \mathrm{~kW}$ y utilizando el refrigerante R134a como fluido de trabajo. El área total de colectores fue $5.6 \mathrm{~m}^{2}$. Se realizó una evaluación experimental para la obtención de los resultados generando un COP entre 1.9 y 2.9, mientras que la temperatura en el tanque fue de $51^{\circ} \mathrm{C}$. Los resultados obtenidos de forma experimental indican que, a una temperatura de evaporación determinada, se puede calcular la potencia del compresor y la potencia transferida por el condensador a una temperatura de evaporación. Los autores indican que el COP en el sistema propuesto se encuentra en el rango de 1.7 a 3.3 para temperaturas de evaporación de -15 a $20^{\circ} \mathrm{C}$.

Mohanraj, Belyayev, Jayaraj y Kaltayev (2018) realizaron un estudio sobre el desarrollo y aplicaciones de los sistemas de bomba de calor asistidas por energía solar. Se analizaron los dife- 
rentes sistemas de bombas de calor, entre los cuales se destacan los sistemas DX-SAHP, geotérmicos, los sistemas convencionales SAHP y sistemas fotovoltaicos híbridos. Un análisis del ciclo de vida de los equipos fue presentado, además de las aplicaciones que se pueden realizar con este tipo de sistemas, secado, calentamiento de espacios, calentamiento de agua y desalinización. Los autores finalizan indicando que la integración de las fuentes renovables de energía con los sistemas de bomba de calor permite la reducción del consumo de fuentes fósiles y sus efectos ambientales. El diseño de los canales puede afectar la eficiencia del colector, así como el rendimiento del sistema. La configuración Tipo 1 es la forma convencional de los canales.

Sun, Wu, Dai y Wang (2014) investigaron y compararon el rendimiento de un sistema DXSAHP con tres diferentes diseños superficiales, paralelo, geometría fractal en "T" y panel de abejas hexagonal, mostrados en la Figura 7. El diseño hexagonal en forma de panel de abejas fue el que maximizó la temperatura de evaporación. Este diseño mejora el COP y la capacidad de calentamiento en un 20.5 y 23.5\%, respectivamente, con respecto a la configuración 1.

Figura 7. Configuraciones de los canales en la parte superior del colector (Sun, Wu, Dai y Wang, 2014)

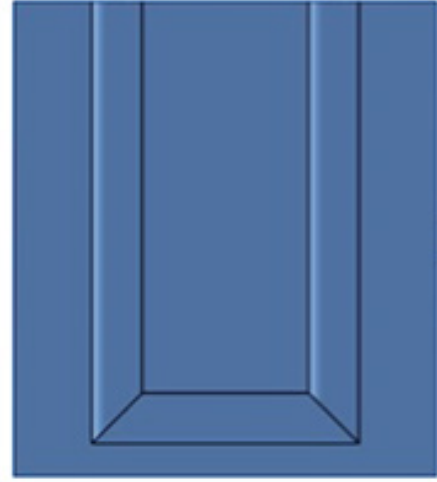

Tipo I

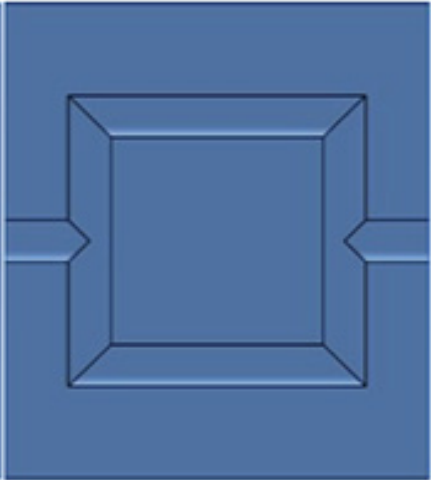

Tipo ॥

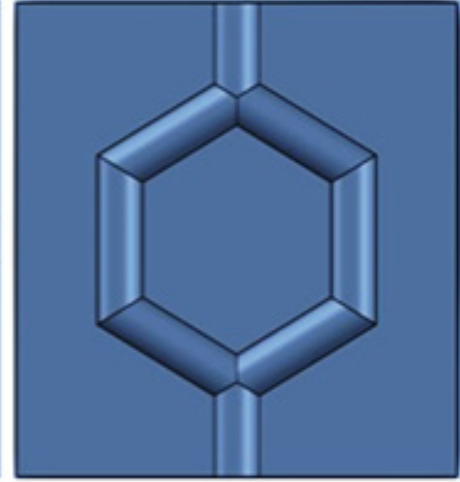

Tipo III

Un dispositivo de expansión termostática con un orificio de diámetro 1.6 mm, una presión de trabajo de 300 MPa y un número de vueltas de 10, fue utilizado en la investigación de Combariza, González y Castilla (2018). Estudiaron una bomba de calor asistida por energía solar utilizando como fluido de trabajo el refrigerante R134a, un compresor de velocidad nominal el cual trabaja a 3500 rpm, un colector/evaporador de placa plana con canales de diámetro interno de $7.74 \mathrm{~mm}$, una longitud total de $15 \mathrm{~m}$, el espesor de la aleta es de $0.5 \mathrm{~mm}$ y la distancia entre tubos es 100 mm. Los resultados obtenidos indican una variación de la temperatura del agua de 25 a $32{ }^{\circ} \mathrm{C}$ luego de 4 minutos aproximadamente y un COP de 2.4. Los autores manifiestan que el bajo COP obtenido se produce por la gran pérdida de presión en el evaporador el cual es de tubos horizontales.

\section{Refrigerantes}

Para utilizar un refrigerante en un determinado sistema se analizan ciertos parámetros, como el potencial de calentamiento global (GWP, por sus siglas en inglés) y el potencial de agotamiento del ozono (ODP, por sus siglas en inglés) (Harby, 2017; ACAIRE, 2013; Zhang, Wu, Li y Kong, 2014). 
La norma ISO 817: 2014 establece una clasificación de los refrigerantes basada en datos de toxicidad e inflamabilidad. Según el estándar de seguridad proporcionado por ASHRAE (2019), se clasifican a los refrigerantes en tres grupos que son altamente inflamables, ligeramente inflamables y no inflamables.

Nawaz, Shen, Elatar, Baxter y Abdelaziz (2017) realizaron una investigación con los refrigerantes R1234yf y el R1234ze, los cuales poseen un bajo GWP. Los resultados señalan que ambos refrigerantes pueden ser considerados como sustitutos del R134a (Bolaji y Huan, 2013). Li (2013) planteó una investigación con el objetivo de reemplazar al R22 en los sistemas DXSAHP, puesto que contiene cloro, elemento que agota la capa de ozono. Los resultados obtenidos muestran que el refrigerante R290 es una mejor alternativa al R22. Igualmente, se analizó una mezcla entre los refrigerantes R290 y R22, con mayor porcentaje de R290 y se obtuvo mejores resultados de COP. El análisis experimental se realizó bajo las condiciones climáticas subtropicales típicas, a temperatura media anual de $18^{\circ} \mathrm{C}$ y una radiación solar entre 300 y 800 W/m², además, el COP varía entre 4.4 y 6.5. La Tabla 1 resume diferentes refrigerantes utilizados en bombas de calor.

Tabla 1. Comparación de las propiedades de refrigerantes. (REFECOL, R22, 2014; GASSERVEI, R134a, 2018; GASSERVEI, R290, 2018; GASSERVEI, R404A, 2018; GASSERVEI, R407C, 2018; GASSERVEI, R410A, 2018; GASSERVEI, R600a, 2018)

\begin{tabular}{|l|l|l|l|l|l|l|l|}
\hline & \multicolumn{1}{|c|}{ R22 } & R134a & \multicolumn{1}{|c|}{ R290 } & R404A & R407C & \multicolumn{1}{|l}{ R410A } & R600a \\
\hline Peso Molecular $[\mathrm{kg} / \mathrm{k} \cdot \mathrm{mol}]$ & 86.47 & 102.03 & 44.10 & 97.61 & 86.2 & 72.6 & 58.1 \\
\hline Punto de ebullición normal $\left.{ }^{\circ} \mathrm{C}\right]$ & -40.8 & -26.1 & -42.1 & -46.45 & -43.6 & -51.58 & -11.7 \\
\hline Temperatura crítica $\left[{ }^{\circ} \mathrm{C}\right]$ & 96.15 & 101.1 & 96.7 & 72.07 & 86.74 & 72.13 & 135 \\
\hline Presión crítica $[\mathrm{MPa}]$ & 4.98 & 4.06 & 4.24 & 3.73 & 4.62 & 4.92 & 3.64 \\
\hline ODP & 0.055 & 0 & 0 & 0 & 0 & 0 & 0 \\
\hline GWP $[100$ años $]$ & 1810 & 1430 & $3-11$ & 3922 & 1774 & 2088 & 3 \\
\hline Grupo de seguridad & A1 & A1 & A3 & A1 & A1 & A1 & A3 \\
\hline
\end{tabular}

Estudios elaborados por Zhu, Xie, Zhang y Guan (2013) sobre sistemas DX-SAHP, indican que el refrigerante sufre un cambio de fase de líquido a vapor por la absorción de calor producido por la energía solar incidente. En la investigación se determinó que la temperatura de funcionamiento del colector y la temperatura de evaporación del refrigerante se mantienen constantes y en el rango de bajas temperaturas. De esta manera, se puede obtener mayor eficiencia en el colector y se evita que la bomba de calor sufra daños por el mal clima. Los resultados de radiación solar, temperatura ambiente promedio y velocidad media del viento que inciden sobre un colector/ evaporador de placa sin cubierta y un colector/evaporador de doble placa son 602 $\mathrm{W} / \mathrm{m}^{2}, 2{ }^{\circ} \mathrm{C}, 1 \mathrm{~m} / \mathrm{s}$ y $664.6 \mathrm{~W} / \mathrm{m}^{2}, 23^{\circ} \mathrm{C}, 0.8 \mathrm{~m} / \mathrm{s}$, respectivamente. Además, en función de los datos obtenidos, los autores concluyen que mientras el área del colector incrementa, el COP del sistema también aumenta, pero se reduce la eficiencia del colector y aumenta la carga de trabajo del compresor.

El estudio de diferentes refrigerantes para reemplazar al R12 en un sistema DX-SAHP fue realizado por Gorozabel, Chaturvedi y Almogbel (2005). Los resultados indican que los valores más altos de COP se presentaron al utilizar los refrigerantes R22 y R134a cuando la temperatura del colector osciló entre 0 y $20^{\circ} \mathrm{C}$. Una comparación entre los refrigerantes R410A y R407C, 
indica que el R410A es más eficiente que el R407C o el R404A, pero no como el R134a. El refrigerante R410A produce valores de COP 15 a 20\% más bajos que con el R134a.

Un análisis comparativo de sistemas de refrigeración doméstica utilizando refrigerantes R600a y R134a es realizado Jara e Isaza (2015). Utilizaron compresores de 1/4 y 1/6 HP, obteniendo valores de COP de 2.7 y 3.5 con R600a y R134a, respectivamente, con el compresor de 1/4 HP. El refrigerante R600a, al ser un hidrocarburo, presenta un grado de inflamabilidad. Los autores concluyen que el refrigerante R600a puede reemplazar al R134a en la refrigeración doméstica por varios motivos, como su bajo tiempo de vida en la atmósfera, que es menor a un año, su bajo potencial de calentamiento global, menor a 8 , siendo un refrigerante ideal y amigable con el medio ambiente.

Los refrigerantes R407C y el R410A son una mezcla zeotrópica, los componentes principales del R407C son R32 / R125 / R134a (23/25/52\% en peso). Mientras que para el R410A son R32 / R125 (50/50\% en peso). Una alternativa para mitigar el impacto ambiental producido por el uso de refrigerantes es el uso de HC y HFO, considerados refrigerantes naturales y de bajo GWP (Wu, Hu y Wang, 2018; Nawas, Shen, Elatar, Baxter y Abdelaziz, 2017). Son utilizados como fluidos de trabajo en sistemas de bombas de calor asistidas por energía solar, aunque son flamables. En la investigación realizada por Ghoubali, Byrne y Bazantay (2017), se utilizaron tres tipos de condensadores, siendo mejor el de tipo roll bond con 10 vueltas, área interna de los canales y superficie de intercambio de calor de 0.465 y $0.24 \mathrm{~m}^{2}$, respectivamente, y volumen interno de $730 \mathrm{~cm}^{3}$. El COP estuvo entre 2.4 y 3.20 dependiendo de la carga de refrigerante R290 $\left(\mathrm{CH}_{3} \mathrm{CH}_{2} \mathrm{CH}_{3}\right)$. Con una carga de $250 \mathrm{~g}$, se alcanzó un COP de 3.20 en un tiempo de calentamiento inferior a 7 horas. La temperatura de subenfriado que alcanzó con una carga de $200 \mathrm{~g}$ de refrigerante fue $5 \mathrm{~K}$. Adicionalmente, Shi, Aye, Li y Du (2019) recomiendan verificar los requerimientos de la aplicación para trabajar en un entorno seguro. La Figura 8 es un esquema proporcional de los refrigerantes con los que trabajan los sistemas DX-SAHP.

Figura 8. Refrigerantes para DX-SAHP (Shi, Aye, Li y Du, 2019)

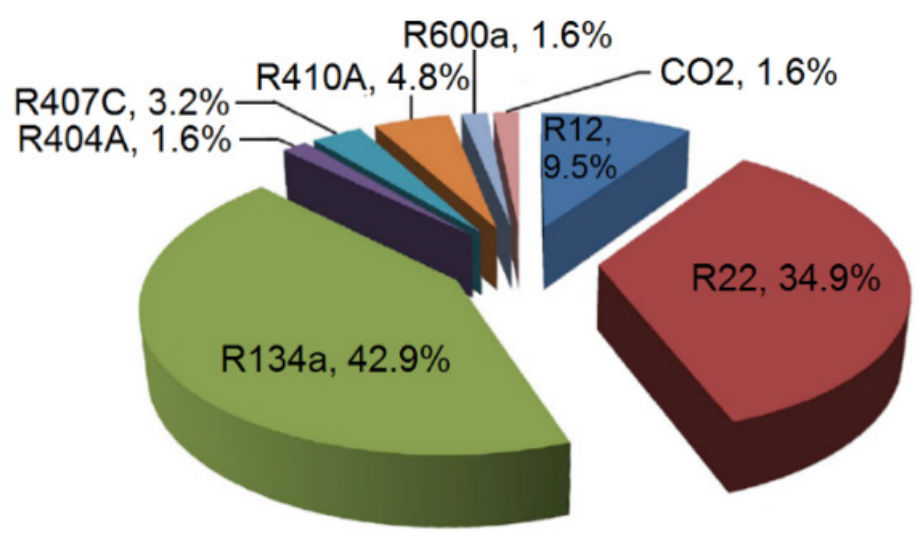

El refrigerante R22 tiene efectos desfavorables para el medio ambiente por lo que no debería ser utilizado. Un estudio realizado por Kasera y Chandra (2017) muestra el uso del refrigerante 407C, un CFC, como reemplazo al R22. Los autores compararon el ODP y el GWP de ambos refrigerantes. Para el refrigerante R22 se presentan valores de 0.055 y 1700, mientras que, para el R407C, 0 y 1530 de ODP y GWP, respectivamente. Además, se comparó los efectos que produ- 
ce el uso de R407C y R22. El COP es menor, la capacidad de enfriamiento es menor y el consumo de energía es mayor. Se debe considerar que el R407C es menos contaminante que el R22 y los autores sugieren alternativas de refrigerantes como reemplazo al R22, mencionando al R134a y propano (R290), considerado como un refrigerante natural.

\section{Coeficiente de rendimiento}

Un análisis de los diferentes sistemas de bombas de calor asistidas por energía solar fue realizado por Buker y Riffat (2016). En la investigación se demuestra que estos sistemas aumentan significativamente su eficiencia al ser comparados con los sistemas convencionales de calentamiento de agua, para corroborar estos valores se consideró la evaluación del COP. Una bomba de calor de expansión directa con asistencia solar fue analizada en Hong Kong, una ciudad con un clima subtropical húmedo que puede alcanzar una temperatura ambiente aproximada de 29 ${ }^{\circ} \mathrm{C}$. Los resultados proporcionados por Chow et al. (2010) indican que se presenta una velocidad del viento promedio anual de $3.08 \mathrm{~m} / \mathrm{s}$ y $1365 \mathrm{~kW} \cdot \mathrm{h} / \mathrm{m}^{2}$ año de radiación solar, con las mencionadas condiciones climáticas el coeficiente de rendimiento promedio anual fue de 6.46 , además de presentar una potencia de ingreso al compresor anual de $1501 \mathrm{~kW} \cdot \mathrm{h}$.

Para la obtención de agua caliente sanitaria, Deng y Yu (2016) desarrollaron un calentador de agua que utiliza energía solar y eólica. El sistema puede ser utilizado cuando la radiación solar es lo suficientemente alta como para calentar el fluido de trabajo que en esta oportunidad fue el refrigerante R134a. Las condiciones de operación del sistema dual fueron, radiación solar entre 100 y $700 \mathrm{~W} / \mathrm{m}^{2}$, temperatura ambiente en el rango entre 10 y $30^{\circ} \mathrm{C}$. La velocidad del compresor fue de $2980 \mathrm{rpm}$, un área de colector de $2 \mathrm{~m}^{2}$, el volumen del tanque de agua caliente fue de 150 litros. Los resultados indican que el COP aumenta en un 14\% aproximadamente al incrementarse la radiación.

Mohamed, Riffat y Omer (2017) analizaron la influencia de factores como la temperatura exterior, radiación solar y temperatura inicial del agua en el rendimiento de una bomba de calor asistida por energía solar. Los resultados indican que con una radiación solar de $200 \mathrm{~W} / \mathrm{m}^{2}$ y una temperatura ambiente de $19.8^{\circ} \mathrm{C}$, se alcanzó un COP de 3.99. Mientras que reduciendo la radiación a $100 \mathrm{~W} / \mathrm{m}^{2}$ y con una temperatura ambiente de $20.1^{\circ} \mathrm{C}$, se obtuvo un COP de 3.87 . El colector solar utilizado se encuentra recubierto con pintura negra y está formado por dos placas de aluminio de $2 \mathrm{~mm}$ de espesor, la tubería es de aluminio de $13 \mathrm{~mm}$ de diámetro. Además, se corrobora que cuando la temperatura del agua en el tanque del condensador aumenta con el tiempo, la temperatura de condensación también aumenta. Los autores indican que el rendimiento del sistema está significativamente influenciado por el área de colector y de la radiación solar.

Yousefi y Moradali (2015) realizaron un análisis termodinámico de una bomba de calor asistida por energía solar considerando parámetros como la radiación solar y el área del colector/evaporador, así como el aumento de temperatura del agua desde 20 a $45^{\circ} \mathrm{C}$. Se instaló un colector/evaporador de $3 \mathrm{~m}^{2}$ de superficie de tipo placa plana con cubierta, además de un tanque para el almacenamiento de agua de $0.150 \mathrm{~m}^{3}$. Los resultados muestran la obtención de un COP de 6.37 a una temperatura ambiente de $15^{\circ} \mathrm{C}$ y radiación de $450 \mathrm{~W} / \mathrm{m}^{2}$. La Figura 9 presenta la variación del COP con relación a la temperatura ambiente y con relación al tiempo necesario para el calentamiento. 
Figura 9. COP en función de la a. temperatura ambiente b. tiempo
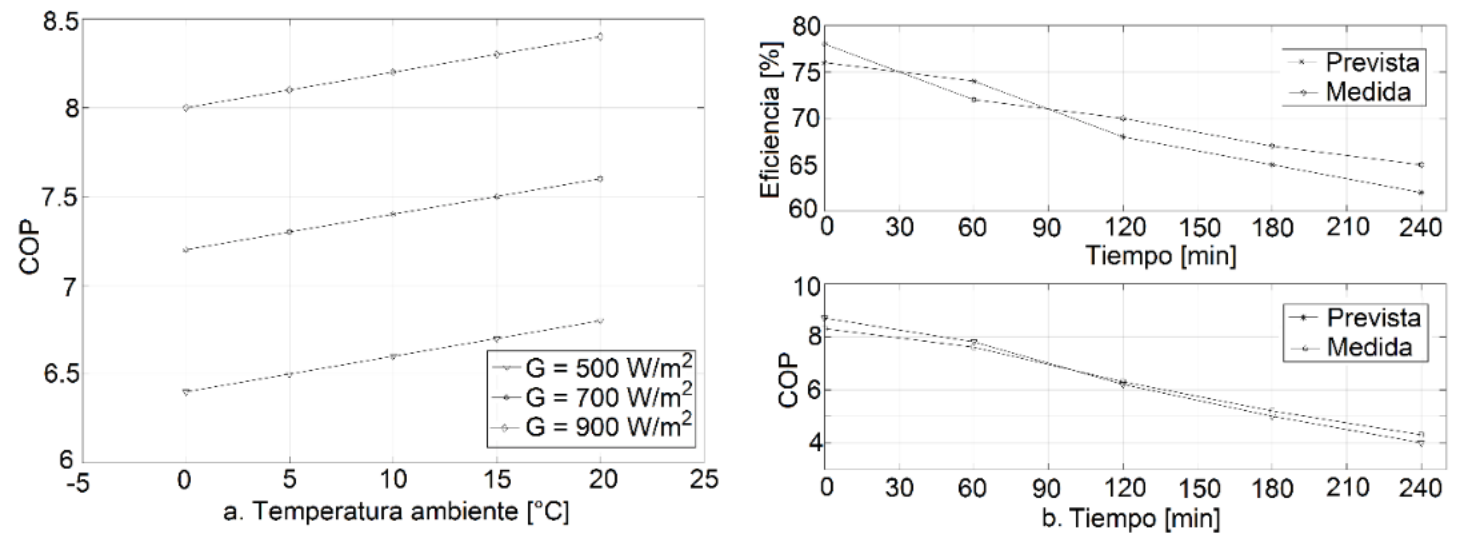

A continuación, en la Tabla 2 se presentan investigaciones exclusivamente para calentamiento de agua, mediante el uso de un sistema de bomba de calor asistida por energía solar, además se proporciona información detallada sobre sus componentes, se explican los diferentes parámetros de funcionamiento como la temperatura del agua, área del evaporador, consumo de energía, refrigerantes utilizados, temperaturas inicial y final del agua, entre otros.

Tabla 2. Análisis de los componentes de los sistemas DX-SAHP

\begin{tabular}{|c|c|c|c|c|c|}
\hline & 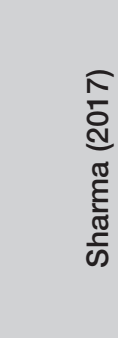 & 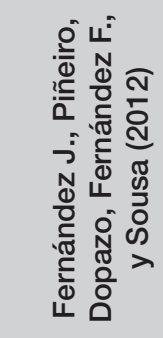 & 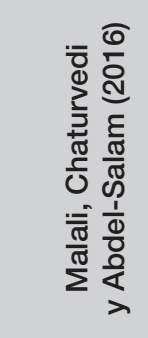 & 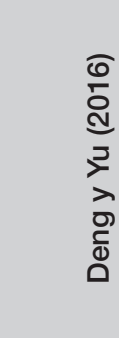 & 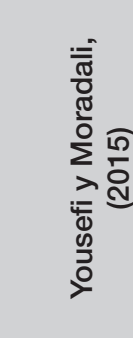 \\
\hline $\mathrm{T}_{\text {inicial }}$ del agua $\left[{ }^{\circ} \mathrm{C}\right]$ & 29 & 14 & 20 & 20 & 20 \\
\hline $\mathrm{T}_{\text {final }}$ del agua $\left[{ }^{\circ} \mathrm{C}\right]$ & 49 & 55 & 50 & 55 & 45 \\
\hline Volumen del tanque $\left(\mathrm{m}^{3}\right)$ & 0.03 & 0.3 & 0.150 & 0.150 & 0.150 \\
\hline Área del colector $\left[\mathrm{m}^{2}\right]$ & 2.23 & 3.23 & 3.7 & 2 & 3 \\
\hline $\mathrm{COP}$ & 4 & 4.29 & 3.6 & 3.91 & 6.37 \\
\hline Consumo energía [kW·h] & 0.245 & 4.31 & & & 0.468 \\
\hline Refrigerante utilizado & R134a & $\mathrm{R} 134 \mathrm{a}$ & R134a & R134a & R134a \\
\hline País & India & España & EE. UU. & China & Irán \\
\hline
\end{tabular}

\section{Conclusiones}

Investigaciones relacionadas al uso de bombas de calor asistidas por energía solar se han presentado en este documento. El principal objetivo es tratar que, mediante el uso de la energía 
solar y la modificación de varios componentes del sistema, por ejemplo, el uso de un evaporador y un colector en una sola unidad, se pueda aumentar el rendimiento del equipo. Así se obtiene una mejora en la eficiencia energética del sistema, además de mitigar el impacto ambiental al proponer nuevas opciones de refrigerantes amigables con el medio ambiente que contribuyen a un desarrollo sustentable.

Algunos problemas que se suscitan en los colectores de agua, la corrosión, congelación nocturna, incrustaciones provocadas por el carbonato de calcio, se eliminan al utilizar como fluido de trabajo un refrigerante. Esto genera un aumento en la vida útil del equipo. Al ser utilizado un refrigerante como fluido de trabajo, circulando dentro del colector/evaporador, la temperatura necesaria para producir la evaporación es baja. Así se consigue pérdidas menores del sistema puesto que el valor de las pérdidas en el colector es una función de la diferencia de temperatura ambiente y la del colector. El colector solar de placa plana sin cubierta trabaja con valores de eficiencia altos y lo único que utiliza es la diferencia de temperaturas que se produce con el ambiente.

Los refrigerantes con un bajo potencial de calentamiento global y bajo potencial de agotamiento del ozono se deben promover en la industria de la refrigeración. Estos refrigerantes presentan buenas características termodinámicas y son el reemplazo ideal para los hidroclorofluorocarbonos, hidrofluorocarbonos y los clorofluorocarbonos que son sustancias que agotan la capa de ozono y además, fomentan el calentamiento global. Un buen reemplazo para estas sustancias son los hidrocarburos, como el R290 y el R600a.

Los paneles solares fotovoltaicos pueden presentar varias aplicaciones, una de ellas es generar la energía eléctrica requerida por el compresor de velocidad variable, uno de los componentes principales del sistema. La eficiencia global en los sistemas DX-SAHP aumenta considerablemente al ser comparados con los sistemas SAHP, puesto que el colector solar funciona como evaporador en el sistema. De esta forma, se evita el uso de una mayor cantidad de partes en el equipo, además de generar un ahorro económico. Los resultados muestran una comparación entre los sistemas DX-SAHP con los sistemas de calentamiento de agua que utilizan un quemador de gas. La energía primaria ahorrada al utilizar un sistema DX-SAHP es aproximadamente del 50 $\%$ bajo las mismas condiciones de operación. Este porcentaje de ahorro es casi independiente del tipo de panel solar de placa plana utilizado. La tecnología utilizada en los sistemas DX-SAHP presenta datos prometedores en diversos escenarios, tanto en invierno, como verano.

Actualmente la cantidad de energía consumida a nivel mundial, que es proveniente de fuentes fósiles es una problemática que perjudica el medio ambiente. Los sistemas DX-SAHP presentan una cantidad reducida de emisiones de gases de efecto invernadero a la atmósfera. Además, utilizan fuentes renovables de energía, por lo tanto, resultan ser la mejor opción para reducir el impacto ambiental causado por el uso de combustibles fósiles.

\section{Agradecimientos}

Este trabajo investigativo tuvo el apoyo del Área de Investigación y Desarrollo de Ingeniería Mecánica de la Universidad Politécnica Salesiana y de la Universidad Pontificia Bolivariana de Medellín. 


\section{Bibliografía}

ACAIRE. (2013). Refrigerantes de Hidrocarburos. Bogotá.

Aguilar, F., Aledo, S., y Quiles, P. (2016). Experimental study of the solar photovoltaic contribution for the domestic hot water production with heat pumps in dwellings. Applied Thermal Engineering(101), 379-389.

ASHRAE. (28 de Mayo de 2019). Safety Standard for Refrigeration Systems. Obtenido de https://webcache.googleusercontent.com/search?q=cache:CLE84mzAxtIJ:https://www.ashrae.org/ File\%2520Library/docLib/StdsAddenda/15_2016_f_20170207.pdf+ycd=3yhl=esyct=clnkyclient $=$ firefox $-b$

Bauer, A., y Menrad, K. (2019). Standing up for the Paris Agreement: Do global climate targets influence individuals' greenhouse gas emissions? Environmental Science and Policy, 99, 72-79.

Benavides, D., Jurado, F., y González, L. (2018). Data analysis and tools applied to modeling and simulation of a PV system in Ecuador. Enfoque UTE, 9, 1-12.

Bolaji, B., y Huan, Z. (2013). Ozone depletion and global warming: Case for the use of natural refrigerant -a review. Renewable and Sustainable Energy Reviews(18), 49-54.

Buker, M., y Riffat, S. (2016). Solar assisted heat pump systems for low temperature water heating applications: A systematic review. Renewable and Sustainable Energy Reviews(55), 399-413.

Cai, J., Ji, J., Wang, Y., y Huang, W. (2016). Numerical simulation and experimental validation of indirect expansion solar-assisted multi-functional heat pump. Renewable Energy(93), 280-290.

Cai, J., Li, Z., Ji, J., y Zhou, F. (2019). Performance analysis of a novel air source hybrid solar assisted pump. Renewable Energy, 139, 1133-1145.

Calise, F., Dentice D`Accadia, M., Figaj, R., y Vanoli, L. (2016). A novel solar-assisted heat pump driven by photovoltaic/thermal collectors: Dynamic simulation and thermoeconomic optimization. Energy(95), 346-366.

Cengel, Y., y Boles, M. (2015). Thermodynamics An Engineering Approach. New York: McGraw-Hill Education.

Cerit, E., y Erbay, B. (2013). Investigation of the effect of rollbond evaporator design on the performance of direct expansion heat pump experimentally. Energy Conversion and Management(72), 163-170.

Charters, W., y Taylor, L. (1976). Some performance characteristics of a solar boosted heat pump, in:Proceedings of the IIR Conference Towards and Ideal Food Chain. Refrigerant Science Technology, 64, 1-8.

Chow, T., Pei, G., Fong, K., Lin, Z., Chan, A., y He, M. (2010). Modeling and application of direct-expansion solarassisted heat pump for water heating in subtropical Hong Kong. Applied Energy(87), 643-649.

Colangelo, G., Favale, E., Miglietta, P., y De Risi, A. (2016). Innovation in flat solar thermal collectors: A review of the last ten years experimental results. Renewable and Sustainable Energy Reviews(57), 1141-1159.

Combariza, H., González, P., y Castilla, C. (2018). Numerical simulation and experimental validation of a solar-assisted heat pump system for heating residential water. International Journal of Refrigeration(86), 28-39.

Deng, W., y Yu, J. (2016). Simulation analysis on dynamic performance of a combined solar/air dual source heat pump water heater. Energy Conversion and Management(120), 378-387.

Duarte, W., Paulino, T., Pabon, J., Sawalha, S., y Machado, L. (2019). Refrigerants selection for a direct expansion solar assisted heat pump for domestic hot water. Solar Energy, 184, 527-538.

Fernández, J., Piñeiro, C., Dopazo, A., Fernández, F., y Sousa, P. (2012). Experimental analysis of a direct expansion solar assisted heat pump with integral storage tank for domestic water heating under zero solar radiation conditions. Energy Conversion and Management(59), 1-8.

Gadea, M., y Gonzalo, J. (2019). Trends in distributional characteristics: Existence of global warming. Journal of Econometrics, 1-22. 
Gallo , C., Faccilongo, N., y La Sala, P. (2017). Clustering analysis of environmental emissions: A study on Kyoto Protocol's impact on member countries. Journal of Cleaner Production, 1-19.

GASSERVEI. (08 de Diciembre de 2018). Ficha técnica R134a. Recuperado el 24 de 01 de 2018, de https:// www.gas-servei.com/images/Ficha_tecnica_R134A.pdf

GASSERVEI. (27 de Febrero de 2019). Ficha técnica R290 (Propano). Obtenido de https://www.gas-servei. com/images/Ficha-tecnica-R290.pdf

GASSERVEI. (27 de Febrero de 2019). Ficha técnica R404A. Obtenido de https://www.gas-servei.com/ images/Ficha-tecnica-R404A.pdf

GASSERVEI. (27 de Febrero de 2019). Ficha técnica R407C. Obtenido de https://www.gas-servei.com/images/Ficha-tecnica-R407C.pdf

GASSERVEI. (27 de Febrero de 2019). Ficha técnica R410A. Obtenido de https://www.gas-servei.com/images/Ficha-tecnica-R410A.pdf

GASSERVEI. (27 de Febrero de 2019). Ficha técnica R600a (Isobutano). Obtenido de https://www.gasservei.com/images/Ficha_tecnica_R600A.pdf

Ghoubali, R., Byrne, P., y Bazantay, F. (2017). Refrigerant charge optimisation for propane heat pump water heaters. International Journal of Refrigeration(76), 230-244.

Gorozabel, F., y Carbonell, T. (2016). Actualidad y perspectivas de una bomba de calor de expansión directa con energía solar. Ingeniería Mecánica, XIX(1), 49-58.

Gorozabel, F., Chaturvedi, S., y Almogbel, A. (2005). Analysis of a direct expansion solar assisted heat pump using different refrigerants. Energy Conversion y Management(46), 2614 - 2624.

Haceb. (25 de Agosto de 2019). Calentadores de agua que funcionan con gas. Obtenido de http://www. haceb.com/LinkClick.aspx?fileticket=UOHWcEOxdEA\%3dyportalid=0

Harby, K. (2017). Hydrocarbons and their mixtures as alternatives to environmental unfriendly halogenated refrigerants: An updated overview. Renewable and Sustainable Energy Reviews(73), 1247-1264.

Homecenter. (14 de Marzo de 2019). Calentador de paso eléctrico. Obtenido de http://www.homecenter. com.co/homecenter-co/product/137832/Calentador-de-paso-electrico-110v-5.5-kW/137832

Hossain, M., Saidur, R., Rahim, N., Islam, M., Ahamed, J., y Rahman, M. (2011). Review on solar water heater collector and thermal energy performance of circulating pipe. Renewable and Sustainable Energy Reviews(15), 3801-3812.

Huang, W., Ji, J., Xu, N., y Li, G. (2016). Frosting characteristics and heating performance of a direct-expansion solar-assisted heat pump for space heating under frosting conditions. Applied Energy(171), 656-666.

International Energy Agency. (03 de Agosto de 2019). World: Indicators. Obtenido de https://www.iea.org/ statistics/statisticssearch/report/?country=WORLDyproduct=indicatorsyyear $=2014$

Jara, N., e Isaza, C. (2015). Análisis comparativo de sistemas de refrigeración doméstica utilizando refrigerantes R600a y R134a. I+T+C Investigación, TEcnología y Ciencia, 1-15.

Junkers. (27 de Julio de 2019). Bombas de calor de agua caliente sanitaria. Obtenido de https://junkerses.resource.bosch.com/media/documentacion/agua_caliente/folletos_comerciales/2017_1/ ficha_supraeco_swo_swi_v1.pdf

Kalogirou, S. (2014). Solar energy engineering. Burlington: Elsevier.

Kannan, N., y Vakeesan, D. (2016). Solar energy for future world: - A review. Renewable and Sustainable Energy Reviews(62), 1092-1105.

Kasera, S., y Chandra, S. (2017). Performance of R407C as an alternate to R22: A review. Energy Proce$\operatorname{dia}(109), 4-10$.

Kong, X., Li, Y., Lin, L., y Yang, Y. (2017). Modeling evaluation of a direct-expansion solar-assisted heat pump water heater using R410A. International Journal of Refrigeration(76), 136-146.

Krockenberger, K., DeGrove, J., Hutzel, W., y Foreman, C. (2014). International High Performance Buildings. Design of a Heat Pump Assisted Solar Thermal System. Indiana. 
Kuang, Y., y Wang, R. (2006). Performance of a multi-functional direct-expansion solar assisted heat pump system. Solar Energy, 80, 795-803.

Li, H. (2013). Study on Alternative Refrigerants for Direct Expansion Solar Assisted Heat Pump System. Applied Mechanics and Materials, 361, 267-270.

Maamoun, N. (2019). The Kyoto protocol: Empirical evidence of a hidden success. Journal of Environmental Economics and Management, 95, 227-256.

Malali, P., Chaturvedi, S., y Abdel-Salam, T. (2016). An approximate method for prediction of thermal performance of direct expansion-solar assisted heat pump (DX-SAHP) systems for water heating applications. Energy Conversion and Management(127), 416-423.

Ministerio del Ambiente. (30 de Julio de 2019). Protocolo de Kioto (pK). Obtenido de http://www.minambiente.gov.co/index.php/component/content/article/458-plantilla-cambio-climatico-14

Miyamoto, M., y Takeuchi, K. (2019). Climate agreement and technology diffusion: Impact of the Kyoto Protocol on international patent applications for renewable energy technologies. Energy Policy, 129, 1331-1338.

Mohamed, E., Riffat, S., y Omer, S. (2017). Low-temperature solar-plate-assisted heat pump: A developed design for domestic applications in cold climate. International Journal of Refrigeration(81), 134-150.

Mohanraj, M., Belyayev, Y., Jayaraj, S., y Kaltayev, A. (2018). Research and developments on solar assisted compression heat pump systems - A comprehensive review (Part A: Modeling and modifications). Renewable and Sustainable Energy Reviews(83), 90-123.

Moreno-Rodriguez, A., Garcia-Hernando, N., González-Gil, A., e Izquierdo, M. (2013). Experimental validation of a theoretical model for a direct-expansion solar-assisted heat pump applied to heating. Energy(60), 243-253.

Nawas, K., Shen, B., Elatar , A., Baxter , V., y Abdelaziz, O. (2017). R290 (propane) and R600a (isobutane) as natural refrigerants for residential heat pump water heaters. Applied Thermal Engineering(127), 870-833.

Nawaz, K., Shen, B., Elatar, A., Baxter, V., y Abdelaziz, O. (2017). R-1234yf and R-1234ze(E) as low-GWP refrigerants for residential heat pump water heaters. International Journal of Refrigeration.

Pandey, K., y Chaurasiya, R. (2017). A review on analysis and development of solar flat plate collector. Renewable and Sustainable Energy Reviews(67), 641-650.

Ravi, P., Krishnaiah, A., Akella, S., y Azizuddin, M. (2015). Evaluation Of Inside Heat Transfer Coefficient of Roll Bond Evaporator for Room Air Conditioner. International Journal of Innovative Research in Science, Engineering and Technology, IV, 3378-3384.

REFECOL. (06 de Junio de 2019). Ficha técnica R22. Recuperado el 20 de 11 de 2017, de http://www.refecol.com.ec/wp-content/uploads/2014/06/FichaTecnica-R22.pdf

Secretaría Nacional de Planificación y Desarrollo. (2013). Plan Nacional de Desarrollo / Plan Nacional para el Buen Vivir 2013 - 2017. Quito.

Sharma, A. (2017). An investigation on comparison of direct expansion solar assisted heat pump water heater with an air-source heat pump water heater. International Journal of Latest Engineering and Management Research, II(3), 44-50.

Sheu, E., y Mitsos, A. (2013). Optimization of a hybrid solar-fossil fuel plant: Solar steam reforming of methane in a combined cycle. Energy, 51, 193-202.

Shi, G.-H., Aye, L., Li, D., y Du, X.-J. (2019). Recent advances in direct expansion solar assisted heat pump systems: A review. Renewable and Sustainable Energy Reviews, 109, 349-366.

Sporn, P., y Ambrose, E. (1955). Proceedings of the world symposium on applied solar energy. The heat pump and solar energy. Phoenix.

Sun, X., Wu, J., Dai, Y., y Wang, R. (2014). Experimental study on roll-bond collector/evaporator with optimized-channel used in direct expansion solar assisted heat pump water heating system. Applied Thermal Engineering(66), 571-579. 
Tagliafico, L., Scarpa, F., y Valsuani, F. (2014). Direct expansion solar assisted heat pumps e A clean steady state approach for overall performance analysis. Applied Thermal Engineering(66), 216-226.

Vargas, J., Medina, J., Pozo, M., Avila, E., Pozo, N., y Salazar, G. (2019). Análisis del uso de micro convertidores DC/DC enfocados en la extracción máxima de energía en una granja fotovoltaica. Enfoque UTE, 10, 205-217.

Velasco, L., Goyos, L., Delgado, R., y Freire, L. (2016). Instalación para medición de conductividad térmica en composites basados en residuos de biomasa. Enfoque UTE, 7, 69-81.

Wang, R., y Ge, T. (2016). Advances in solar heating and cooling. Cambridge: Elsevier Ltd.

Wu, D., Hu, B., y Wang, R. (2018). Performance simulation and exergy analysis of a hybrid source heat pump system with low GWP refrigerants. Renewable Energy(116), 775-785.

Yousefi, M., y Moradali, M. (2015). Thermodynamic analysis of a direct expansion solar assisted heat pump water heater. Journal of Energy in Southern Africa, XXVI(2), 110-117.

Zhang, D., Wu, Q., Li, J., y Kong, X. (2014). Effects of refrigerant charge and structural parameters on the performance of a direct-expansion solar-assisted heat pump system. Applied Thermal Engineering(73), 522-528.

Zhou, X., y Feng, C. (2016). The impact of environmental regulation on fossil energy consumption in China: Direct and indirect effects. Cleaner Production.

Zhu, M., Xie, H., Zhang, B., y Guan, X. (2013). The Characteristics of the Evaporator/Evaporator for Direct Expansion Solar Assisted Heat Pump System. Journal of Power and Energy Engineering(1), 73-76. 